Rev. salud pública. 13 (4): 703-716, 2011

Revisión/Review

\title{
Acolhimento no Programa Saúde da Família: revisão das abordagens em periódicos brasileiros
}

The relationship between healthcare personnel and patients (user acceptance) regarding the family healthcare program: a review of approaches in Brazilian journals

Ialane Monique Vieira dos Santos e Adriano Maia dos Santos

Instituto Multidisciplinar em Saúde, Campus Anísio Teixeira, Universidade Federal da Bahia. Vitória da Conquista, Bahia, Brasil. ialanesantos@hotmail.com, maiaufba@ufba.br

Recebido em 26 Janeiro 2011/Enviado para Modificação 31 Julio 2011/Aprovado 15 Agosto 2011

\section{RESUMO}

Trata-se de uma revisão sistemática de literatura acerca do tema "Acolhimento no Programa de Saúde da Família-PSF" com o objetivo de identificar e analisar as diferentes abordagens em estudos selecionados em periódicos brasileiros. Utilizouse a Biblioteca Virtual em Saúde a partir do argumento de busca "acolhimento" AND "saúde da família", perfazendo, no final da busca e dos refinamentos, um escopo de 39 artigos. Os estudos foram sistematizados segundo as dimensões do acolhimento no PSF. Compreenderam-se as dimensões do acolhimento em cinco categorias: 1. Acolhimento: desafios à produção do cuidado; 2. Acolhimento: práticas inovadoras que fortalecem o cuidado no PSF; 3. Acolhimento: dispositivo para a consolidação da integralidade; 4. Acolhimento: ação produtora de educação em saúde, adesão terapêutica e qualidade de vida; 5. Acolhimento: inclusão social, dignidade e respeito. $\mathrm{O}$ acolhimento evidencia-se enquanto uma tecnologia leve de grande impacto na promoção à saúde que estreita o vínculo, fortalece o PSF, mobiliza a sensibilidade dos trabalhadores da saúde, requerendo uma ação reflexiva, o desenvolvimento ético e solidário para escutar e dialogar, gerando satisfação dos profissionais e dos usuários. O acolhimento possibilita que o PSF torne-se a porta de entrada preferencial, contribuindo significativamente para construção e consolidação dos princípios do Sistema Único de Saúde.

Palavras-chave: Programa Saúde da Família, acolhimento, atenção primária à saúde, assistência à saúde, serviços de saúde, sistema único de saúde, sistemas nacionais de saúde (fonte: DeCS, BIREME).

\section{ABSTRACT}

This literature review was aimed at identifying and examining approaches to healthcare personnel's relationships with patients (user acceptance/reciprocal 
understanding) regarding the family health program (FHP) in selected studies in Brazilian journals. The virtual health library (biblioteca virtual em saúde - BVS) was searched, using the terms "user acceptance" (acolhimento) AND "family health" (saúde da família), giving 39 papers by the end of the search and its refinements. The studies were systematized by considering "user acceptance" in the FHP. User acceptance/reciprocal understanding fell into five categories: challenges when providing healthcare, innovative practices strengthening FHP healthcare, devices for consolidating reciprocal understanding, action producing health education, adhesion to therapy and quality of life, and social inclusion, dignity and respect. Health personnel's relationships with patients was manifest as a high-impact, soft technology approach for promoting healthcare by strengthening bonds. It strengthened the FHP, promoted health workers' sensitivity, requiring reflective action and the development of ethics and solidarity to listen to each other and promote dialogue, thereby generating satisfaction among healthcare service personnel and users. Reciprocal understanding enables the FHP to become the preferential gateway for providing healthcare and thus contribute significantly towards constructing and consolidating the principles of Brazil's national health system, the unified healthcare system (Sistema Único de Saúde).

Key Words: Family healthcare program, user acceptance, primary health care, health care delivery, health service, unified health system, national health system (source: $\mathrm{MeSH}, \mathrm{NLM}$ ).

\section{RESUMEN}

Aceptación en el programa salud de la familia: repaso de los enfoques en revistas brasileñas

Se trata de un repaso de la literatura sobre el tema "Aceptación en el Programa de Salud de la Familia - PSF" con el objetivo de identificar y analizar los diferentes enfoques de los estudios seleccionados en periódicos brasileños. Se utilizó la Biblioteca Virtual en Salud a partir del argumento de búsqueda "acolhimento" (aceptación) AND "saúde da família" (salud de la familia), llegando, al final de la búsqueda y del perfeccionamiento, a un alcance de 39 artículos. Los estudios se organizaron teniendo en cuenta las dimensiones de aceptación en el PSF. Se consideraron las dimensiones del aceptación dentro de cinco categorías (1)Aceptación: desafíos en la producción del cuidado; (2) Aceptación: prácticas innovadoras que fortalecen el cuidado en el PSF; (3) Aceptación: dispositivo para la consolidación de la integralidad; (4) Aceptación: acción productora de educación en salud, adhesión terapéutica y calidad de vida; (5) Aceptación: inclusión social, dignidad y respeto. El aceptación surge como una tecnología simple de gran impacto en la promoción de la salud que estrecha el vínculo. Fortalece el PSF, moviliza la sensibilidad de los trabajadores de la salud, lo que requiere una acción reflexiva, el desarrollo ético y solidario para escuchar y dialogar, que conllevan la satisfacción de los profesionales y de los usuarios. El aceptación permite que el PSF se vuelva puerta de entrada principal, lo que contribuye significativamente a la construcción y consolidación de los principios del Sistema Único de Saúde (Sistema Único de Salud).

Palabras Clave: Programa de Salud Familiar, aceptación, atención primaria de salud, prestación de atención de salud, servicios de salud, sistema único de salud, sistemas nacionales de salud (fuente: DeCS, BIREME). 
$\mathrm{O}$ Programa de Saúde da Família (PSF) surge como estratégia proposta pelo Ministério da Saúde, em 1994, para superar o modelo assistencial centrado em procedimentos e de perfil hospitalar, apontados como responsáveis pela "ineficiência do setor; insatisfação da população; desqualificação profissional; iniqüidades" (1). Busca fortalecer a atenção básica, a partir da descentralização da assistência para o local mais próximo em que vivem as pessoas, pautando-se na integralidade e humanização dos cuidados (2).

Desse modo, necessita de equipes multiprofissionais, com práticas interdisciplinares e políticas intersetoriais articuladas, para dar conta dos complexos problemas de saúde (3). Um dos dispositivos propostos tem sido o acolhimento, para enfrentamento e resolução dos problemas de saúde demandados, com ênfase na renovação e no fortalecimento de vínculos, coresponsabilidade e confiança entre profissionais e a comunidade (4).

Os problemas no âmbito do PSF são agravados pelo modelo de formação, quando não instiga nos futuros profissionais um novo olhar, condizente e apropriado para a atuação no sistema público de saúde. Assim, para o cuidado integral, é patente compreender as necessidades coletivas e individuais no campo biológico, econômico, educacional, social e ambiental com propósito de contemplar os determinantes e condicionantes da saúde-doença. Percebese, dentro dessa lógica, um modelo de atenção que amplia os cenários de atuação para distintos pontos do contexto social, não restringindo o cuidado à unidade de saúde e à intervenção clínica (5).

O acolhimento é uma diretriz operacional que parte dos seguintes princípios: "1. Atender todas as pessoas que procuram os serviços de saúde, garantindo a acessibilidade universal. Assim, o serviço de saúde assume sua função precípua, a de acolher, escutar e dar uma resposta positiva, capaz de resolver os problemas de saúde da população. 2. Reorganizar o processo de trabalho, a fim de que este desloque seu eixo central, do médico para uma equipe multiprofissional - equipe de acolhimento-, que se encarrega da escuta do usuário, comprometendo-se a resolver seu problema de saúde. 3. Qualificar a relação trabalhador-usuário, que deve se dar por parâmetros humanitários, de solidariedade e cidadania" (6).

O atual modelo de atenção à saúde demanda esforços, compromisso e criatividade para implementação do acolhimento, sendo fundamental integrar trabalhador e usuário, os múltiplos saberes, conciliando as diferenças, com a 
finalidade de incluir a voz do outro (7). Nesse sentido, "acolher é, pois, encontrar outra forma de lidar com a diferença. O contato com o diferente é a possibilidade de aprender algo novo, é a possibilidade real de expandir meu mundo" (8).

O trabalho no PSF requer um novo perfil para os profissionais e novos olhares para a compreensão ampliada das necessidades de saúde da população. Nota-se que essa proposta é prejudicada em relação à formação profissional quando sobrevaloriza a prática hospitalar e o profissional médico, em detrimento da atenção básica e da produção do cuidado (9).

Compreende-se como imprescindível intervir na formação profissional para ampliar as estratégias de promoção da saúde, estimular e fortalecer a atenção básica, pois, o saber biomédico desarticulado da atenção básica observado como predominante na formação e atuação de muitos profissionais da saúde - revela-se insuficiente e danoso (10).

O acolhimento, no PSF, desvela-se como uma ação fundamental para a política de humanização da atenção à saúde, pois possibilita priorizar as situações de maior vulnerabilidade biológicas, econômicas e social (11). O acolhimento potencializa o processo de educação em saúde, do exercício e da construção da cidadania, produzindo ações usuário-centradas (12).

A percepção de acolhimento tem se caracterizado por uma dimensão restrita à triagem administrativa e repasse de encaminhamentos, ou ainda, a recepção burocrática, sem uma adequada avaliação do risco, agravo e grau de sofrimento explícito ou velado, ações essas que muitas vezes acentuam uma prática excludente $(8,11,13)$. Quando isso ocorre, descaracteriza-se a atenção do PSF, que se torna inadequada para a produção da integralidade, adesão à terapêutica e da prevenção e controle do adoecimento.

Diante da importância do acolhimento nas práticas em saúde, o estudo tem como objetivo: revisar e analisar as produções em periódicos brasileiros que tratam do tema acolhimento no PSF, buscando identificar as diferentes abordagens em estudos selecionados.

\section{METODOLOGIA}

Trata-se de uma revisão de literatura acerca do tema "Acolhimento no Programa de Saúde da Família". Utilizou-se a Biblioteca Virtual em Saúde 
(BVS) (http://regional.bvsalud.org) com o recurso da metapesquisa, optandose pelo "método de pesquisa integrado", a partir do argumento de busca "acolhimento" AND "saúde da família", identificando-se 126 referências. Para refinar a busca foram selecionados na área de clusters os agrupamentos texto completo AND tipo artigo AND idioma português -, sendo encontradas 60 referências. Entre os artigos selecionados como relevantes para a pesquisa, após o refinamento, um total de 21 artigos foram excluídos, pois cinco apresentaram-se em duplicidade e outros 16 artigos não abordavam assuntos referentes ao PSF, perfazendo, no final da busca, um escopo de 39 artigos.

Segundo as dimensões do acolhimento no PSF, os artigos foram agrupados em cinco categorias (Tabela 1), sendo que um mesmo artigo pode compor mais de uma categoria: (I) Acolhimento: desafios para a produção do cuidado; (II) Acolhimento: práticas inovadoras que fortalecem o cuidado no PSF; (III) Acolhimento: dispositivo para a consolidação da integralidade; (IV) Acolhimento: ação produtora de educação em saúde, adesão terapêutica e qualidade de vida; (V) Acolhimento: inclusão social, dignidade e respeito.

A análise compreendeu uma etapa exploratória com o levantamento dos artigos relacionados ao objetivo da pesquisa, que após seleção circunscreveu um espaço temporal de dez anos (2001-2010). A segunda etapa constituiu na organização do material com leitura flutuante e posterior leitura exaustiva para identificar as estruturas relevantes nos textos. Na terceira etapa, organizaram-se os artigos segundo as categorias que emergiram, conformando a discussão dos diferentes estudos.

Tabela 1. Categorias de análise

\begin{tabular}{ll}
\hline \multicolumn{1}{c}{ Categorias } & Artigos relacionados \\
\hline Acolhimento: desafios para produção do cuidado & $14-39$ \\
Acolhimento: práticas inovadoras que fortalecem o & $14,40-43$ \\
cuidado no PSF & $26,38,44,45$ \\
$\begin{array}{l}\text { Acolhimento: dispositivo para a consolidação da } \\
\text { integralidade }\end{array}$ & $35,41,47-51$ \\
$\begin{array}{l}\text { Acolhimento: ação produtora de educação em saúde, } \\
\text { adesão terapêutica e qualidade de vida }\end{array}$ & $22,31,33,35,38,40,45,48,50,5$ \\
Acolhimento: inclusão social, dignidade e respeito & 2,53 \\
\hline
\end{tabular}




\section{RESULTADOS}

Acolhimento: desafios para Produção do Cuidado

Estudos (14-39) que abordam entraves organizacionais nas práticas que limitam o desempenho de uma atenção qualificada para resolução dos problemas de saúde, desafiando a implantação do acolhimento. Foram agrupados como: limites para atender de forma apropriada às demandas (14-27); infra-estrutura inadequada e ausência de projetos de ambiência $(15,16,18,22,28,29)$; desarticulação entre os diferentes níveis de atenção e fragilidade do PSF para coordenar os cuidados (14-17,21,23,24,26,30-32); cuidado fragmentado, centrado na medicalização e em tecnologias duras $(15,16,18,21-26,29,30,32-34)$; falta de preparo para lidar no PSF $(14,16,17,25,32,34-36)$; ruídos na comunicação entre os trabalhadores $(16,17,23,35)$; dificuldade para penetrar nas redes sociais (16,33); restrição de insumos $(22,24,26,29)$; fragmentação no sistema de informação (37) e entraves na adoção do acolhimento (15-18,22$24,26,27,29,33,35,38,39)$.

Os artigos abordam questões inerentes à baixa cobertura, histórica insuficiência de políticas sociais, busca por procedimentos e desvalorização das ações educativas e a medicalização da sociedade, como elementos que impactam na população e a fazem buscar os serviços de saúde, sobrecarregando a porta de entrada.

A infra-estrutura inadequada e ausência de projetos de ambiência são evidenciadas em estudos que apontam essas questões como entraves à escuta qualificada, à acomodação dos usuários e profissionais, à privacidade, ou seja, impactam nos processos de acolhimento, pois constrangem os processos comunicacionais.

Outra questão comum foi a reprodução de cuidados fragmentados, centrados na medicalização e em tecnologias duras. Os trabalhadores permanecem atuando nas equipes de maneira individualizada, tentando resolver os problemas centrados em atos clínicos e prescritivos, limitando os atendimentos à ordem de chegada, solicitando exames complementares, nem sempre, baseados na necessidade do usuário.

Essas questões apontam uma série de implicações à implantação do acolhimento, ao mesmo tempo são consequência de uma série de outras questões: falta de preparo de trabalhadores para lidar com questões complexas; 
educação em serviço muito teórica e desarticulada das necessidades reais; dificuldade na realização de trabalhos interdisciplinares, gerando disputas dentro das equipes; dificuldade de penetração nas redes sociais de apoio a cuidados prolongados, principalmente aos mais vulneráveis e socialmente discriminados, que requerem a co-responsabilidade da própria comunidade.

Acolhimento: práticas inovadoras que fortalecem o cuidado no PSF

Sintetiza alguns relatos de experiências de acolhimento (14,40-43), que discutem como essa tecnologia relacional pode ajudar a superar adversidades encontradas no contexto de produção do cuidado, nas equipes de PSF.

Os artigos relatam a redução da tensão nas portas de entrada das unidades de saúde $(40,41,43)$, construção de novos projetos terapêuticos com a inclusão dos usuários $(14,40-42)$, articulação entre diferentes serviços (CAPS-USF) (14), fortalecimento da relação interdisciplinar (43), criação de materiais educativos e abordagem educativa personalizada para idosos portadores de diabetes (41) e o acolhimento a grupos de travestis (42).

A inovação de práticas no cotidiano torna-se vital para reconhecimento do PSF como espaço de produção do cuidado. $\mathrm{O}$ acolhimento implantado em um PSF criou um espaço terapêutico para conversa, interação e integração de trabalhadores e usuários (40). Scholze et al. (43) descrevem que o acolhimento em uma USF buscava ouvir a demanda dos usuários e fazer os encaminhamentos adequados para os profissionais da própria unidade ou para serviços de referência.

Outra estratégia inovadora relata a experiência de articulação entre CAPS e PSF que amplia e enriquece a rede de acolhimento, pautando-se na integração, mediada por reuniões em equipe, capacitações, estudos de casos e visitas domiciliares. Um terceiro relato apresenta como inovação no acolhimento a ênfase dada a sua potencialidade educacional, de empoderamento e autonomia do usuário para o autocuidado e coresponsabilização no projeto de saúde. A ação inovadora deu-se na postura dos trabalhadores envolvidos no processo de escuta qualificada, no cuidado e preparo rigoroso de materiais educativos e instrumentos personalizados para as demandas singulares dos idosos portadores de diabetes.

Uma quarta experiência relata a invenção de formas alternativas para promoção do acolhimento às travestis, que apresentavam distanciamento quanto 
à busca de assistência à saúde no PSF. Os profissionais romperam com a clínica tradicional e passaram a visitar as travestis nas suas residências, realizando consultas, promoveram atividades educativas com a comunidade $\mathrm{e}$ produção de material educativo específico (42).

Acolhimento: dispositivo para consolidação da integralidade

Os quatro artigos $(26,38,44,45)$ apresentam a potência do acolhimento como dispositivo necessário para consolidação do princípio da integralidade. Segundo Cecílio (46), há uma dimensão denominada de "integralidade focalizada", que leva em conta tudo que pode ser feito dentro de um estabelecimento/equipe para resolver os problemas de saúde. Toda USF deve disponibilizar a máxima potência para acolher as demandas, escutando e fazendo encaminhamentos adequados, pois, "a integralidade deve ser fruto do esforço e confluência dos vários saberes de uma equipe multiprofissional, no espaço concreto e singular dos serviços de saúde, sejam eles um centro de saúde, uma equipe de PSF ou um hospital" (46).

Gomes e Pinheiro (38) mostram a necessidade de superar o reducionismo biológico a partir da escuta, da conversa qualificada e compreensão das necessidades de saúde para além do explícito fisicamente, com o propósito de responder adequadamente, de forma integrada e integral, fortalecendo também as ações de prevenção.

Outro artigo (45) expõe a política de humanização do SUS como uma estratégia de construção de espaços de negociação para viabilizar a clínica ampliada para contribuir e viabilizar a consolidação da integralidade. Propõe processos de educação com gestores e profissionais, com o objetivo de multiplicar o número de apoiadores institucionais comprometidos com a proposta de capilarizar o acolhimento em toda a rede de serviços (45).

Nascimento, Correia e Nozawa (44) relatam a experiência de Campinas, destacando a implantação do modelo Paidéia, como reorganizador das práticas em toda rede, tendo o acolhimento como atributo estruturante.

Por fim, Souza et al. (26) destacam o acolhimento, enquanto um processo imprescindível para construção do cuidado integral no PSF, pois "amplia vínculos e melhora a compreensão sobre as necessidades dos usuários". 
Acolhimento: produtor de educação em saúde, adesão terapêutica e qualidade de vida

Os sete artigos $(35,41,47-51)$ evidenciam o fortalecimento dos vínculos de confiança, apoio mútuo, interação e integração entre profissionais que compõem a equipe e usuários, com produção de satisfação, qualidade da assistência e qualidade de vida, com repercussões na compreensão do processo saúdedoença e mudanças de comportamentos de risco.

O acolhimento enquanto ferramenta para o processo de educação em saúde revela-se enquanto uma prática de educação solidária e emancipatória, no sentido do exercício e da construção da cidadania, pela escuta e troca de saberes entre conhecimento técnico e popular (12).

Ao fortalecer o vínculo de confiança, o acolhimento permitiu a abertura da privacidade espontânea de algumas mulheres portadoras de HIV/AIDS que optam por revelar esse diagnóstico ao trabalhador da saúde, a partir de atividades educativas que respeitavam a autonomia das usuárias. As ações acolhedoras das equipes asseguravam o sigilo, ajudando no aumento da adesão ao tratamento e práticas sexuais seguras para prevenção de DST/AIDS (48).

Dois estudos argumentam que a participação em algum grupo de educação em saúde aumenta a adesão ao tratamento não farmacológico em portadores de diabetes mellitus, melhorando inclusive o autocuidado $(41,49)$. Outros artigos sustentam, também, que os grupos de saúde, o apoio social e a educação em saúde são formas de acolhimento que podem construir, aprimorar e intensificar vínculos, bem como motivar os sujeitos para a adesão ao tratamento, aquisição de habilidades para o autocuidado e mudanças de comportamentos $(35,47,50,51)$.

Acolhimento: inclusão social, dignidade e respeito

Os artigos abordam as potencialidades do acolhimento para a inclusão social do usuário na dinâmica de trabalho e na construção do próprio projeto de saúde, na perspectiva de inclusão social, dignidadee respeito $(22,31,33,35,38,40,45,48,50,52,53)$. Destacam o rompimento com a lógica médico-centrada, a construção de relações interdisciplinares entre profissionais e relações horizontais com os usuários. 
O PSF pode ser um espaço para elaborar ações na comunidade voltadas para o apoio social e redução de estigmas, preconceitos e discriminação. Essas ações podem ser desenvolvidas no PSF e mediadas por redes sociais sensibilizadas e motivadas para conhecer, aceitar e respeitar as diferenças, valorizar e apoiar portadores de transtornos mentais $(33,52,53)$, portadores de HIV (48), entre outros agravos.

Estudo realizado em contexto hospitalar (52) aborda trabalho desenvolvido em acompanhamentos terapêuticos e sua potência para atuar na prevenção de crises e reinternações hospitalares. Os resultados apontam dificuldades de inclusão dos portadores de transtornos mentais no contexto familiar, social e institucional, evidenciando, assim, a desconexão da rede social com esses sujeitos, bem como a carência de dispositivos de acolhimento substitutivos ao modelo manicomial.

Um relato de experiência aborda a implantação de espaços para o acolhimento coletivo (40), por todos os profissionais que compõem o PSF. Trata-se de uma inovação que privilegia a escuta qualificada, as relações interdisciplinares e horizontais, a circulação do saber, experiências e aspirações, surgindo nesse meio a discussão acerca dos mais variados assuntos e demandas de saúde da comunidade, bem como variadas respostas e construções de projetos terapêuticos com a inclusão do usuário.

Gomes e Pinheiro (37) relacionam o acolhimento com as práticas desenvolvidas no PSF em grandes centros, identificando essa estratégia como elemento para integralidade e estabelecimento do vínculo, na construção da saúde como direito. Outros estudos defendem a formação de espaços coletivos que incluam as necessidades dos usuários, tornando o PSF a porta de entrada preferencial dos usuários do SUS $(35,50)$.

Guedes, Pitombo e Barros (45) apresentam o processo de formação na política de humanização do SUS, enquanto uma proposta de capilarização de intencionalidades e ações para garantia dos direitos de saúde e dignidade dos usuários. Esse processo de formação foi dirigido aos profissionais e gestores, buscando o apoio desses sujeitos, para multiplicar as propostas de acolhimento nas práticas, na perspectiva da humanização da saúde.

Outro artigo apresenta a realização de grupos vivenciais nas escolas com familiares de crianças do ensino fundamental, mostrando que olhares e ações ampliadas de acolhimento aos sujeitos, considerando o contexto social e familiar, 
produzem respeito e inclusão social, que geram melhorias na compreensão do processo saúde-doença, nas relações, melhorando a resolução de problemas no núcleo familiar com repercussão na saúde mental e dignidade dos sujeitos (53).

Rabello e Rodrigues (31) tratam de cuidados paliativos infantis realizados por um programa de assistência interdisciplinar domiciliar. Destacam que ações articuladas de atenção básica com a rede podem favorecer a permanência das crianças no contexto familiar e social em que vivem, com amenização de sofrimentos, agravos, insatisfação e estresse de familiares.

\section{Considerações Finais}

Os estudos mostram o acolhimento como dispositivo carreado de potencialidades para produzir práticas inovadoras que fortalecem o cuidado, consolidam a integralidade, qualificam as atividades de educação em saúde, aumentando a adesão terapêutica e a inclusão social.

Para tanto, o acolhimento deverá mudar radicalmente o processo de trabalho em saúde (6), mas precisará ser um projeto permanentemente estimulado como estratégia de gestão, por meio da educação permanente, melhoria nas condições de trabalho, adequação dos equipamentos de saúde, debate e apoio dos conselhos locais e municipais, ou seja, o acolhimento precisará ser um processo de reorientação na produção dos cuidados $(4,54)$.

O acolhimento fortalece o PSF, pois mobiliza a sensibilidade dos profissionais, requerendo uma ação reflexiva, desenvolvimento ético e solidário para escutar e dialogar, recuperando o sentido da clínica e colocando as pessoas em primeiro lugar, contribuindo significativamente para construção e consolidação dos princípios do SUS

\section{REFERÊNCIAS}

1. Franco TB, Merhy EE. Programa de Saúde da Família (PSF): contradições de um programa destinado à mudança do modelo tecnoassistencial. In: Merhy EE, Magalhães Júnior HM, Rimoli J, Franco TB, Bueno WS (orgs.). O trabalho em saúde: olhando e experienciando o SUS no cotidiano. São Paulo: Hucitec; 2003. p.55-124.

2. Brasil. Portaria $n^{\circ} 648$, de 28 de Março de 2006. Política Nacional de Atenção Básica. Poder executivo, Brasília, DF; 28 Mar, 2006.

3. Silva IZQJ, Trad LAB. O trabalho em equipe no PSF: investigando a articulação técnica e a interação entre os profissionais. Interface comunic. saúde educ. 2004; 9(16):25-38. 
4. Panizzi M, Franco TB. A implantação do Acolher Chapecó Reorganizando o Processo de Trabalho. In:Franco TB, Peres MAA, Foschiera MMP, Panizzi M (orgs.). Acolher Chapecó: uma experiência de mudança do modelo assistencial, com base no processo de trabalho. São Paulo: Hucitec; 2004. p.79-110.

5. Campos WS. Saúde Paidéia. São Paulo: Hucitec; 2003.

6. Franco T, Bueno WS, Merhy EE. O acolhimento e os processos de trabalho em saúde: o caso de Betim. In: Merhy EE, Magalhães Júnior HM, Rimoli J, Franco TB, Bueno WS (orgs.). O trabalho em saúde: olhando e experienciando o SUS no cotidiano. São Paulo: Hicitec; 2003. p.37-54.

7. Teixeira RR. O Acolhimento num Serviço de Saúde Entendido como uma Rede de Conversações. In: Pinheiro R, Mattos RA. Construção da Integralidade: cotidiano saberes e práticas em saúde. $4^{a}$ Edição. Rio de Janeiro: IMS-UERJ/Abrasco; 2007. p. 91-113.

8. São Paulo. Acolhimento: o pensar, o fazer, o viver. São Paulo: Secretaria Municipal de São Paulo; 2002.

9. Santos AM, Kochergin CN, Jesus SR, Soares DA, Assis MMA, Bispo Júnior JP, et al. Linhas do cuidado e responsabilização no PSF de um município da região Sudoeste da Bahia: um olhar analisador. In: Assis MMA, Nascimento MAA, Franco TB, Jorge MSB (orgs.). Produção do cuidado no Programa Saúde da Família: olhares analisadores em diferentes cenários. Salvador: Edufba; 2010. p.59-82.

10. Cunha GT. A construção da clínica ampliada na atenção básica. São Paulo: Hucitec; 2005. Brasil. Acolhimento nas práticas de produção de saúde. $2^{\text {a }}$ Edição. Brasília: Ministério da Saúde; 2008.

11. Traesel CA, Bedin FNP, Gallina LS, Daneluz SFC, Santos AC, Melo TT, et al. Educação em saúde: fortalecendo a autonomização do usuário. In: Franco TB, Peres MAA, Foschiera MMP, Panizzi M (orgs.). Acolher Chapecó: uma experiência de mudança do modelo assistencial, com base no processo de trabalho. São Paulo: Hucitec; 2004. p.111-142.

12. Brasil. Política Nacional de Humanização: acolhimento com avaliação e classificação de risco: um paradigma ético-estético no fazer em saúde. Brasília: Ministério da Saúde; 2004.

13. Delfini PSS, Sato MT, Antoneli PP, Guimarães POS. Parceria entre CAPS e PSF: o desafio da construção de um novo saber. Ciênc. saúde coletiva 2009; 14 (Supl.1):1483-1492.

14. Coelho MO, Jorge MSB, Araújo ME. O acesso por meio do acolhimento na atenção básica à saúde. Rev. baiana saúde pública 2009: 33 (3):440-452.

15. Coelho MO, Jorge MSB. Tecnologia das relações como dispositivo do atendimento humanizado na atenção básica à saúde na perspectiva do acesso, do acolhimento e do vínculo. Ciênc. saúde coletiva 2009; 14 (Supl.1):1523-1531.

16. Cunha ABO, Vieira-da-Silva LM. Acessibilidade aos serviços de saúde em um município do Estado da Bahia, Brasil, em gestão plena do sistema. Cad Saude Publica 2010; 26 (4):725-737.

17.Fracolli LA, Zoboli ELCP. Descrição e análise do acolhimento: uma contribuição para o Programa de Saúde da Família. Rev Esc Enferm USP 2004; 38 (2):143-151.

18. Nascimento PTA, Tesser CD, Poli Neto P. Implantação do acolhimento em uma unidade local de saúde de Florianópolis. ACM arq. catarin. med 2008; 37(4):32-34.

19. Nery SR, Nunes EFPA, Carvalho BG, Melchior R, Baduy RS, Lima JVC. Acolhimento no cotidiano dos auxiliares de enfermagem nas Unidades de Saúde da Família, Londrina (PR). Ciênc. saúde coletiva 2009; 14 (5):1411-1419.

20. Rocha PM, Uchoa AC, Rocha NSPD, Souza ECF, Rocha ML, Pinheiro TXA. Avaliação do Programa Saúde da Família em municípios do Nordeste brasileiro: velhos e novos desafios. Cad Saude Publica. 2008; 24 (Supl.1):S69-S78.

21. Santos AM, Assis MMA, Rodrigues AAAO, Nascimento MAA, Jorge MSB. Linhas de tensões no processo de acolhimento das equipes de saúde bucal do Programa Saúde da Família: o caso de Alagoinhas, Bahia, Brasil. Cad Saude Publica. 2007; 23 (1):75-85.

22. Santos $A M$, Assis MMA. Da fragmentação à integralidade: construindo e (des)construindo a prática de saúde bucal no Programa de Saúde da Família (PSF) de Alagoinhas, BA. Ciênc. saúde coletiva 2006; 11(1):53-61. 
23. Schimith MD, Lima MADS. Acolhimento e vínculo em uma equipe do Programa Saúde da Família. Cad Saude Publica. 2004; 20(6):1487-1494.

24. SilvaACMA, Villar MAM, Cardoso MHCA, Wuillaume SM. Aestratégia saúde da família: motivação, preparo e trabalho segundo médicos que atuam em três distritos do município de Duque de Caxias, Rio de Janeiro, Brasil. Saúde Soc. 2010; 19(1):159-169.

25. Souza ECF, Vilar RLA, Rocha NSPD, Uchoa AC, Rocha PM. Acesso e acolhimento na atenção básica: uma análise da percepção dos usuários e profissionais de saúde. Cad Saude Publica. 2008; 24(Supl.1):S100-S110.

26. Takemoto MLS, Silva EM. Acolhimento e transformações no processo de trabalho de enfermagem em unidades básicas de saúde de Campinas, São Paulo, Brasil. Cad Saude Publica. 2007; 23(2):331-340.

27. Pina JC, Mello DF, Mishima SM, Lunardelo SR. Contribuições da estratégia Atenção Integrada às Doenças Prevalentes na Infância ao acolhimento de crianças menores de cinco anos. Acta paul. enferm. 2009; 22(2):142-148.

28. Rodrigues MP, Lima KC, Roncalli AG. A representação social do cuidado no programa saúde da família na cidade de Natal. Ciênc. saúde coletiva. 2008; 13 (1):71-82.

29. Pinafo E, Lima JVC, Baduy RS. Acolhimento: concepção dos auxiliares de enfermagem e percepção de usuários em uma unidade de saúde da família. Espaç. saúde (Online). 2008; 9(2):17-25.

30. Rabello CAFG, Rodrigues PHA. Saúde da família e cuidados paliativos infantis: ouvindo os familiares de crianças dependentes de tecnologia. Ciênc. saúde coletiva. 2010; 15 (2):379-388.

31. Tanaka OU, Ribeiro EL. Ações de saúde mental na atenção básica: caminho para ampliação da integralidade da atenção. Ciênc. saúde coletiva. 2009; 14(2):477-486.

32. Brêda MZ, Augusto LGS. O cuidado ao portador de transtorno psíquico na atenção básica de saúde. Ciênc. saúde coletiva. 2001; 6 (2):471-480.

33. Vecchia MD, Martins STF. Concepções dos cuidados em saúde mental por uma equipe de saúde da família, em perspectiva histórico-cultural. Ciênc. saúde coletiva. 2009; 14(1):183-193.

34. Oliveira A, Silva Neto JC, Machado MLT, Souza MBB, Feliciano AB, Ogata MN. A comunicação no contexto do acolhimento em uma unidade de saúde da família de São Carlos, SP. Interface comunic. saúde educ. 2008; 12(27):749-762.

35. Ximenes Neto FRG, Costa MCF, Rocha J, Cunha ICKO. Auxiliares e técnicos de enfermagem na saúde da Família: Perfil sociodemográfico e necessidades de qualificação. Trab. educ. saúde. 2008; 6(1):51-64.

36. Baptista EK, Marcon SS, Souza RKT. Avaliação da cobertura assistencial das equipes de saúde da família às pessoas que faleceram por doenças cerebrovasculares em Maringá, Paraná, Brasil. Cad Saude Publica. 2008; 24 (1):225-229.

37. Gomes MCPA, Pinheiro R. Acolhimento e vínculo: práticas de integralidade na gestão do cuidado em saúde em grandes centros urbanos. Interface comun. saúde educ. 2005; 9 (17):287-302

38. Schraiber LB, Oliveira AFPL, França Júnior I, Strake SS, Oliveira EA. A violência contra mulheres: demanda espontânea e busca ativa em Unidade Básica de Saúde. Saúde Soc. 2000; 9 (1/2):3-15.

39. Cavalcante Filho JB, Vasconcelos EMS, Ceccim RB, Gomes LB. Acolhimento coletivo: um desafio instituinte de novas formas de produzir o cuidado. Interface comun. saúde educ. 2009; 13(31):315-328.

40. Hammerschmidt KSA, Lenardt MH. Tecnologia educacional inovadora para o empoderamento junto a idosos com diabetes mellitus. Texto \& contexto enferm. 2010; 19(2):358-365.

41. Romano VF. As travestis no Programa Saúde da Família da Lapa. Saúde Soc. 2008; 17 (2):211-219.

42. Scholze AS, Ávila LH, Silva MM, Dacoreggio STK. A implantação do Acolhimento no processo de trabalho de Equipes de Saúde da Família. Espaç. Saúde (Online). 2006; 8(1):7-12. 
43. Nascimento EPL, Correa CRS, Nozawa MR. O município de Campinas e organização da secretaria municipal de saúde. Rev. ciênc. méd. 2007; 16(3):161-173.

44. Guedes CR, Pitombo LB, Barros MEB. Os processos de formação na Política Nacional de Humanização: a experiência de um curso para gestores e trabalhadores da atenção básica em saúde. Physis (Rio J.). 2009; 19(4):1087-1109.

45. Cecílio LCO. As necessidades de saúde como conceito estruturante na luta pela integralidade e equidade na atenção em saúde. In: Pinheiro R, Mattos RA (orgs.). Os sentidos da integralidade na atenção e no cuidado à saúde. $8^{\mathrm{a}}$ Edição. Rio de Janeiro: Cepesc-IMS/ Uerj-Abrasco; 2009. p.117-130.

46. Duarte SJH, Andrade SMO. Assistência pré-natal no programa saúde da família. Esc. Anna Nery Rev. Enferm. 2006; 10(1):121-126.

47. Abdalla FT, Nichiata LY. A Abertura da privacidade e o sigilo das informações sobre o HIVIAids das mulheres atendidas pelo Programa Saúde da Família no município de São Paulo, Brasil. Saúde Soc. 2008; 17(2):140-152.

48. Assunção TS, Ursine PGS. Estudo de fatores associados à adesão ao tratamento não farmacológico em portadores de diabetes mellitus assistidos pelo Programa Saúde da Família, Ventosa, Belo Horizonte. Ciênc. saúde coletiva. 2008; 13(2):2189-2197.

49. Monteiro MM, Figueiredo VP, Machado MFAS. Formação do vínculo na implantação do Programa Saúde da Família numa Unidade Básica de Saúde. Rev Esc Enferm USP. 2009; 43 (2):357-363.

50. SáLD, Souza KMJ, Nunes MG, Palha PF, Nogueira JA, Villa TCS. Tratamento de tuberculose em Unidades de Saúde da Família: histórias de abandono. Texto \& contexto enferm. 2007; 16(4):712-718.

51. Fiorati RC, Saeki T. O acompanhamento terapêutico na internação hospitalar: inclusão social, resgate de cidadania e respeito à singularidade. Interface comunic. saúde educ. 2008; 12(27):763-772.

52. Macêdo VC, Monteiro ARM. Educação e saúde mental na família: experiência como grupos vivenciais. Texto \& contexto enferm. 2006; 15(2):222-230.

53. Silveira PAF. O Acolher Chapecó. In: Franco TB, Peres MAA, Foschiera MMP, Panizzi M (orgs.). Acolher Chapecó: uma experiência de mudança do modelo assistencial, com base no processo de trabalho. São Paulo: Hucitec; 2004. p.70-78.

54. Medeiros FA, Araújo-Souza GC, Albuquerque-Barbosa AA, Clara-Costa I. Acolhimento em uma Unidade Básica de Saúde: a satisfação do usuário em foco. Rev. salud pública (Bogotá). 2010; 12(3):402-413. 